\title{
Case Presentation: Iatrogenic Psychosis
}

\author{
Fariba $\mathbf{K}^{1 *}$ Garrett $\mathbf{P}^{1}$ Tcheremissine $\mathbf{O V}{ }^{2}$ \\ ${ }^{1}$ University Hospital and Medical Center Program/HCA East Florida, USA \\ ${ }^{2}$ Professor of psychiatry, Carolinas Medical Center, USA
}

\begin{abstract}
Tardive dyskinesia (TD) is a neuromuscular disorder that can precipitate following the administration of dopamine receptor antagonists such as neuroleptics. Withdrawal-emergent dyskinesia (WE-D), a less recognized subtype of TD, can manifest shortly after abrupt cessation of antipsychotic use regardless of the neuroleptic subclass used. Super sensitivity psychosis is another possible symptom of acute withdrawal from neuroleptics that can occur alone or concomitant with WE-D. Because of their ever increasing off-label indications and attenuated extrapyramidal side effect profiles, atypical antipsychotics are becoming ubiquitous in both inpatient and outpatient settings, reflecting their utility. However, it is likely that this trend will also result in a greater prevalence of WE-D and super sensitivity psychosis. Clinicians without expertise in the utilization of neuroleptics are likely to misattribute withdrawal symptoms precipitated by abrupt cessation of antipsychotics with toxicity to these agents and erroneously withhold or delay their reinitiation, thereby exacerbating symptoms and inadvertently prolonging patient distress. This case report will illustrate the unique and perplexing presentation of WE-D and super sensitivity psychosis in an acute setting, and provide an effective diagnostic and treatment approach to resolving these conditions.
\end{abstract}

Keywords: Tardive dyskinesia, WE-D, super sensitivity psychosis

\section{Case Presentation: Iatrogenic Psychosis}

Mrs. X, age 46, with a past psychiatric history of major depressive disorder (MDD) with psychotic features, presents to the emergency department accompanied by her husband following a twoday history of erratic behavior. Upon initial evaluation, the patient is alert but disoriented, disheveled, and restless. Dichotomous motor behavior is observed as involuntary, purposeless, and jerky movements of the head, neck, and mouth vis-à-vis posturing of the bilateral upper and lower extremities. Intermittent protrusion of the tongue and sialorrhea are also noted. Throughout the interview, the patient's gaze is inappropriately fixated, unreactive to stimulation of the visual field by means of hand-waving, and interrupted only by bilateral ocular paroxysms of upward deviation. Mrs. X's mood is indiscernible as speech production is non-spontaneous, limited to incoherent verbigerations, and expressed with a flat affect. Without provocation nor warning, Mrs. X lunges at the provider and grabs him around the neck. Appropriate safety protocols are implemented, and the patient is administered intramuscular injections of haloperidol, diphenhydramine, and alprazolam. Further evaluation of her mental status is limited secondary to the sedative effects of the medications. Vital signs and urine drug screen are unremarkable. Mrs. X is medically cleared and transferred to the psychiatric inpatient unit for further evaluation.

\section{History Continued: Collateral Information}

Collateral information is obtained from the patient's husband who reports the patient was diagnosed with MDD with psychotic features twenty years ago. He states that the diagnosis occurred prior to their union and is unable to offer additional information regarding the circumstances leading to the diagnosis. The husband

\begin{tabular}{|l|l|}
\hline \hline Quick Response Code: & *Corresponding author: Kamron Fariba, D.0., 917 NE 3rd St unit 9, Fort Lauderdale, FL \\
33301, USA & Published: 14 December, 2021 \\
Received: 28 October, 2021 & \\
& Citation: Fariba K, Garrett P, Tcheremissine OV. Case Presentation: Iatrogenic Psychosis. J \\
Psych Sci Res. 2021;1(5):1-4. DOI: 10.53902/JPSSR.2021.01.000522
\end{tabular}


notes that Mrs. $\mathrm{X}$ has remained relatively stable on quetiapine monotherapy for over 20 years with occasional and self-resolving episodes of transient paroxysmal affective perturbations that have not warranted hospitalization. He is unaware of any further hospitalizations following the patient's initial diagnosis, nor has he observed severe side effects from the medication, including dyskinesias. The medication was progressively titrated to the patient's current dose at presentation of $600 \mathrm{mg}$ per day--300mg every morning and night. Of critical importance, Mrs. X had remained medication compliant until 3 days prior to presentation when, for unknown reasons, she abruptly discontinued the quetiapine. Motor and cognitive disturbances manifested approximately 1 day following medication cessation.

\section{Evaluation: Continued Manifestations of Psychosis and Dyskinesia}

Upon transfer to the psychiatric inpatient unit, the patient continues to display a constellation of catatonic, dyskinetic, and disorganized behavior, requiring continuous one-to-one observation. The patient is unable to follow commands, minimally verbal, and unable to maintain ADLs. While the majority of her presentation is consistent with a state of abulia, staff note intermittent paroxysms of action including attempts to disrobe and elope from the room, as well as multiple incidents during which the patient lunged at staff in close proximity. During this observation period, the treatment team continues to observe suspicious ocular dyskinesias as well as catalepsy of bilateral upper extremities.

\section{Multiple Choice Question}

Which of the following phenomena can be ruled out based upon the information provided by the vignette?
a. Oculogyric crisis
b. Hyperkinetic catatonia
c. Neuroleptic malignant syndrome
d. Super sensitivity psychosis

Answer: neuroleptic malignant syndrome--vitals are stable, no autonomic dysfunction, no muscle rigidity

\section{The Authors' Observations}

The unusual constellation of symptoms is what makes this case clinically fascinating and relevant. While the patient is ostensibly experiencing some derivation of a thought disorder, she is also exhibiting symptoms consistent with extrapyramidal side effects (EPS) and catatonia-associated behaviors. The key clinical question is whether the symptoms precipitated as side effects of neuroleptic administration or withdrawal, or are symptomatic of a psychotic episode. In treating the psychosis--via neuroleptic administra- tion--one could expect to exacerbate both the catatonia and EPS, whereas addressing the latter leaves the psychosis unattended. The treatment team concludes it is unlikely that the motor abnormalities are a manifestation of EPS as quetiapine is only associated with a minimal EPS diathesis. Furthermore, the patient has been on the regimen for 20 years and it is unlikely that EPS would develop with sudden onset after such an extensive treatment period with good toleration of the medication. Figure 1 illustrates that EPS is most likely to occur within the first 2 months of neuroleptic initiation, if at all. (9) Moreover, the 6-hour half-life of quetiapine would suggest that the drug had been eliminated 24 hours after cessation as 4-5 half-lives are typically required for $94-97 \%$ of a drug to be fully eliminated from the body or below any clinically meaningful serum concentration. ${ }^{1}$

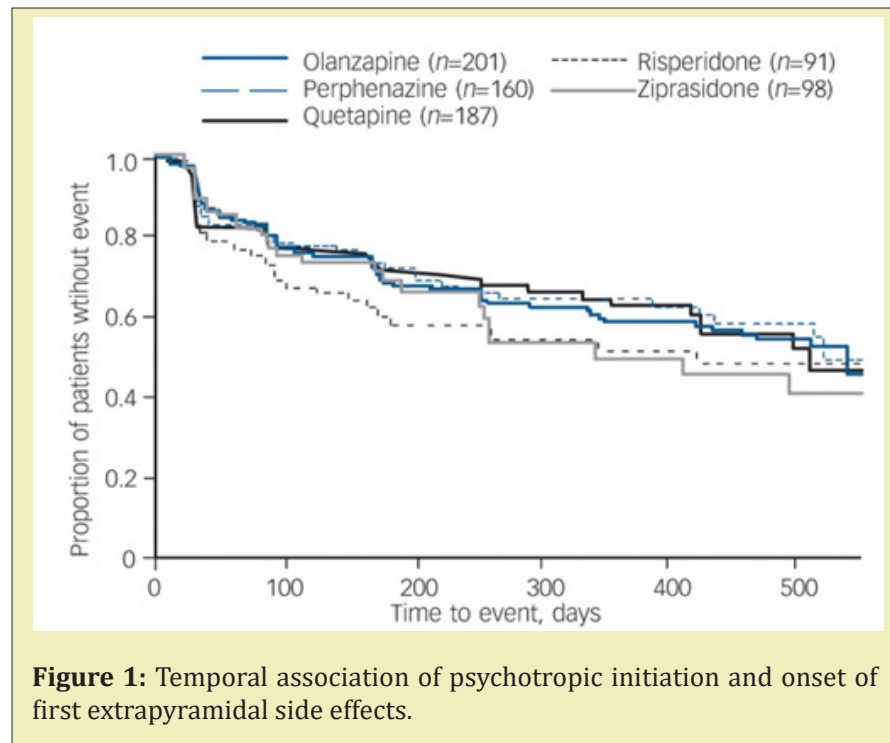

\section{Research: Withdrawal-Emergent Dyskinesia (We-D)}

A review of the literature reveals a few isolated cases of new-onset dyskinesias following abrupt cessation of chronic neuroleptic use. The phenomenon is termed withdrawal emergent dyskinesia (WE-D) and is classified as a subtype of tardive dyskinesia (TD). Additionally, abrupt withdrawal from neuroleptic use can also result in supersensitivity psychosis. ${ }^{2}$ TD is a neuromuscular disorder that can precipitate following the administration of dopamine receptor antagonists. Leading postulations for the etiology of TD attribute dopamine-receptor upregulation, and thus a hypersensitive receptor state, to chronic antipsychotic use. ${ }^{3}$ Acute withdrawal of dopamine antagonism in the setting of already hypersensitized dopamine receptors could theoretically precipitate both phenomena. Locally, functional disturbances to the nigrostriatal and mesolimbic tracts are implicated in manifestations of WE-D and supersensitivity psychoses, respectively. ${ }^{4}$ Furthermore, both are proposed to resolve upon reintroduction of the suspended antipsychotic. 


\section{Treatment: Restarting Quetiapine}

After deliberation, the team decides to restart her quetiapine, and on day 2 of admission, she is restarted on her home regimen of $300 \mathrm{mg}$ twice daily with an almost instantaneous efficacious response. The motor abnormalities are the first to improve. Both the waxy flexibility of the upper extremities and the ocular and neck dystonias resolve by the following day. The patient is taken off one-to-one precautions and downgraded to line-of-sight as she becomes more amenable to direction. Speech production becomes more spontaneous, accompanied by a return of a more congruent affect. The patient's thought process gradually becomes more linear and by day 4 she is responding appropriately to questioning and maintaining ADLs. By day 7, after sufficient return to baseline which is confirmed by the husband, the patient is discharged home.

\section{Discussion: Exigency of Delimiting Between Diagnoses}

The ubiquity of atypical antipsychotics in both inpatient and outpatient settings is primarily a function of their attenuated extrapyramidal side effect (EPS) profiles relative to the typical, first-generation neuroleptics, as well as their increasing off-label indications. Recent studies report TD diathesis rates among first and second-generation neuroleptics to be $30 \%$ and $21 \%$, respectively. ${ }^{5}$ The relative risk reduction seen with the second-generation atypical antipsychotics has led to greater frequencies in both their FDA indicated and off-label uses. Figure 2 demonstrates this trend, specifically in regards to the use of quetiapine. ${ }^{6}$ However, the rise in atypical antipsychotic use has not occurred in conjunction with an equivalent nor sufficient increase in the understanding of this medication class's side effect profile. In an article published in Karger in 2017, Dr. Giovanni Fava, MD suggests that this cavalier prescribing behavior leads to subsequent iatrogenically induced morbidity--what he terms 'iatrogenic comorbidity'. The concept of iatrogenic comorbidity encompasses a number of phenomena including paradoxical reactions, treatment-emergent affective switches, and discontinuation syndromes. Both WE-D and super sensitivity psychosis fall under the umbrella of iatrogenic comorbidity. As previously mentioned, WE-D can develop concurrently, but not exclusively, with super sensitivity psychosis following abrupt cessation of antipsychotic use regardless of the neuroleptic subclass. ${ }^{5}$ Information on these phenomena, including their myriad (and sometimes paradoxical) symptomatic presentations and mechanisms, as well as their acute treatment and management, must be more readily available to the psychiatric community. Such severe outcomes linked to neuroleptics, regardless of their frequency, cannot remain as esoteric knowledge among providers who utilize these medications on a frequent or daily basis. ${ }^{8}$ Equipped with this information, providers will hopefully apply a more thorough and informed benefit-to-harm analysis when prescribing and titrating antipsychotics. Additionally, should WE-D develop, clinicians will be less likely to misattribute this physiological rebound effect to drug toxicity (e.g. EPS) and erroneously withhold or delay antipsychotic reinitiation to obviate the symptoms. WE-D and super sensitivity psychosis may increase in frequency as off-label uses of atypical antipsychotics expand. It is therefore imperative that clinicians be aware of these potentially debilitating sequelae so that medications are not prescribed superfluously and, when necessary, management can be provided promptly and effectively. If discontinuation of an antipsychotic remains a goal despite an episode, WE-D, it is recommended that the medication be restarted and gradually tapered over a period of 1-3 months. ${ }^{3}$ It is also feasible that the 'rebound psychosis' is instead the recurrence of an underlying psychopathology, which would still indicate the reintroduction of the discontinued neuroleptic. ${ }^{9}$

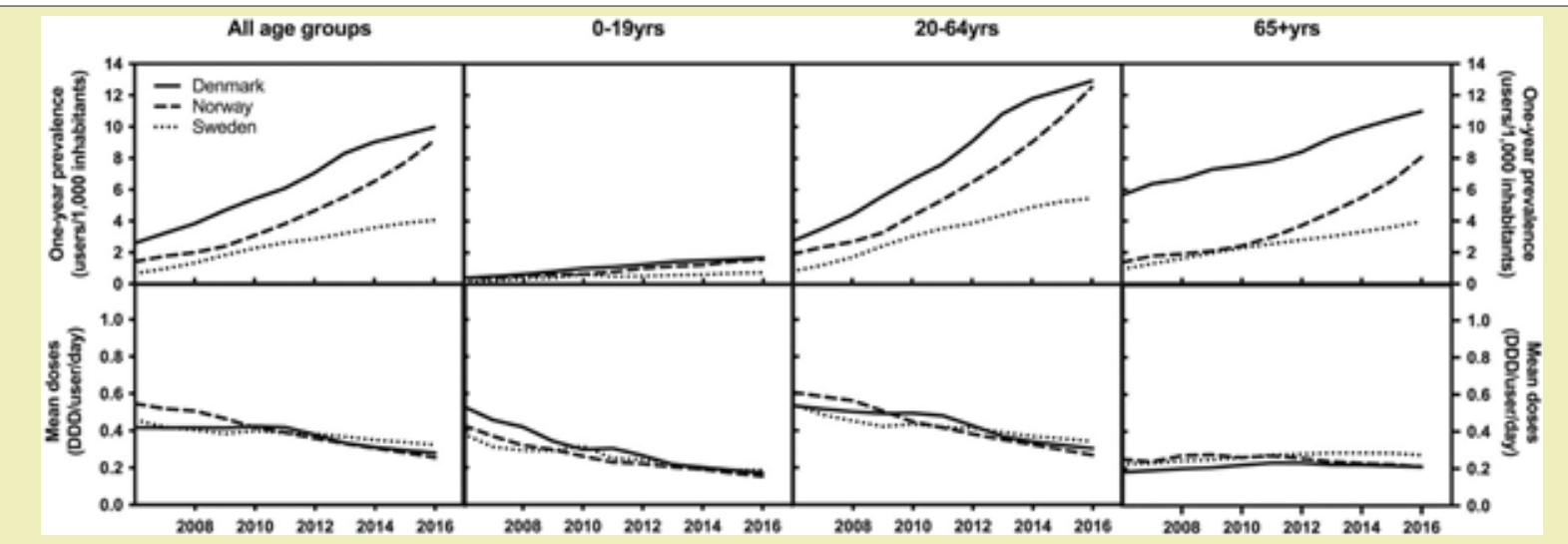

Figure 2: Trends for prevalence (top) and mean doses (bottom) for quetiapine by country from 2006 to 2016.

\section{Outcome: Impotence of Compliance}

Upon discharge, the treatment team educates Mrs. X and her husband regarding her condition and emphasizes the importance of remaining compliant with her medication regimen. The patient and her husband acknowledge the potential sequelae associated with abrupt cessation of quetiapine. She is provided discharge documentation highlighting pertinent information for her reference. 
To date, 6 months following discharge, the patient remains out of the hospital and has exhibited no episodes of psychosis or affective instability.

\section{Bottom Line}

Clinicians must acknowledge the entire spectrum of iatrogenic sequelae when administering psychotropic agents. ${ }^{10}$ Upon initiation of psychotropic medications, the risks associated with abrupt cessation must be made clear to patients. ${ }^{11}$ As well, it is incumbent on psychiatric providers to be able to identify and distinguish between withdrawal and acute intoxication symptoms so that otherwise effective medications are not discontinued. ${ }^{12-15}$

\section{Drug Brand Names}

Quetiapine - Seroquel

\section{Acknowledgments}

None.

\section{Funding}

None.

\section{Conflicts of Interest}

Author declares that there is no conflict of interest.

\section{References}

1. Hallare J, Gerriets V. Half Life. In: StatPearls [Internet]. Treasure Island (FL): Stat Pearls Publishing; 2021.

2. Widschwendter CG, Hofer A. Antipsychotic-induced tardive dyskinesia: update on epidemiology and management. Curr Opin Psychiatry. 2019;32(3):179-184.

3. Karaș H, Güdük M, Saatcioğlu Ö. Withdrawal-Emergent Dyskinesia and Super sensitivity Psychosis Due to Olanzapine Use. Noro Psikiyatr Ars. 2016;53(2):178-180.
4. Chouinard G, Chouinard VA. Atypical antipsychotics: CATIE study, drug-induced movement disorder and resulting iatrogenic psychiatric-like symptoms, super sensitivity rebound psychosis and withdrawal discontinuation syndromes. Psychother Psychosom. 2008;77(2):6977.

5. Waln O, Jankovic J. An update on tardive dyskinesia: from phenomenology to treatment. Tremor Other Hyperkinet Mov (N Y). 2013;3.

6. Højlund M, Pottegård A, Johnsen E, et al. Trends in utilization and dosing of antipsychotic drugs in Scandinavia: Comparison of 2006 and 2016. Br J Clin Pharmacol. 2019;85(7):1598-1606.

7. Miller DD, Caroff SN, Davis SM, et al. Extrapyramidal side-effects of antipsychotics in a randomised trial. Br J Psychiatry. 2008;193(4):279288.

8. Brandt L, Bschor T, Henssler J, et al. Antipsychotic Withdrawal Symptoms: A Systematic Review and Meta-Analysis. Front Psychiatry. 2020;11:569912.

9. Emsley R, Nuamah I, Gopal S, et al. Relapse After Antipsychotic Discontinuation in Schizophrenia as a Withdrawal Phenomenon vs Illness Recurrence: A Post Hoc Analysis of a Randomized Placebo-Controlled Study. J Clin Psychiatry. 2018;79(4):17m11874.

10. Horowitz MA, Jauhar S, Natesan S, et al. A Method for Tapering Antipsychotic Treatment That May Minimize the Risk of Relapse. Schizophr Bull. 2021;47(4):1116-1129.

11. Chouinard G, Samaha AN, Chouinard VA, et al. Antipsychotic-Induced Dopamine Super sensitivity Psychosis: Pharmacology, Criteria, and Therapy. Psychother Psychosom. 2017;86(4):189-219.

12. Sachdev PS. The current status of tardive dyskinesia. Aust N Z J Psychiatry. 2000;34(3):355-369.

13. Ahmad MT, Prakash KM. Reversible hyperkinetic movement disorder associated with quetiapine withdrawal. Mov Disord. 2010;25(9):13081309.

14. Correll CU, Leucht S, Kane JM. Lower risk for tardive dyskinesia associated with second-generation antipsychotics: a systematic review of 1-year studies. Am J Psychiatry. 2004;161(3):414-425.

15. Fava GA, Benasi G, Cosci F. The Potential Role of Iatrogenic Comorbidity in the Interaction between Pharmacotherapy and Psychotherapy in Anxiety Disorders. Verhaltenstherapie. 2017;27:265-270. 